\title{
Controlling Bovine Serum Albumin Release from Biomimetic Calcium Phosphate Coatings
}

\author{
Xiaohua Yu, Mei Wei* \\ Department of Chemical, Materials \& Biomolecular Engineering, University of Connecticut, Storrs, CT, 06269,USA \\ Email:*m.wei@ims.uconn.edu
}

Received November $30^{\text {th }}, 2010$; revised December $20^{\text {th }}, 2010$; accepted December $30^{\text {th }}, 2010$

\begin{abstract}
Biomimetic calcium phosphate (CaP) coating has been used successfully for protein delivery, but the release of protein from CaP coating is mainly dependent on the limited dissolution of the CaP coating and the passive diffusion of the protein in the CaP coating. In the present work, our aim is to improve the release behavior of protein from CaP coating and make it more controllable. By using bovine serum albumin (BSA) as a model protein, our strategy is to tailor BSA release profiles by controlling the distribution of BSA in CaP coatings. To achieve this aim, BSA was added to a modified simulated body fluid ( $m-S B F)$ at different stages of coating formation to obtain tailored BSA release profiles. Sustained BSA release was obtained when BSA was added to $m-S B F$ at the initial stage of the coating where the BSA was incorporated into the lattice structure of the coating. In contrast, a relatively faster release was observed when BSA was added during the later stage of coating formation where BSA was mainly adsorbed to the coating surface. As a result, the BSA release efficiency could be tailored by adding BSA into m-SBF at different coating formation stages. More importantly, the coating composition was not altered with the change of BSA adding times and all the beneficial properties of the biomimetic coating were reserved. Therefore, the BSA release from CaP coatings can be tailored by adjusting its distribution in the coating to achieve a more satisfactory release profile.
\end{abstract}

Keywords: Biomimetic Coating, Apatite, Controlled Release, Release Efficiency

\section{Introduction}

Calcium phosphate (CaP) coating has been applied widely onto titanium implants in orthopedic and dental applications to enhance bone formation around implants and improve clinical success at an early stage after implantation [1,2]. Many methods have been used to produce $\mathrm{CaP}$ coatings on titanium substrate, such as plasma splaying, sputtering deposition, sol-gel coating and biomimetic coating [3-6]. Among these coating techniques, biomimetic coating has attracted more and more attention in the past two decades $[7,8]$. Generally, calcium phosphate is deposited onto a substrate in a simulated body fluid (SBF) which is maintained at a mild temperature and physiological $\mathrm{pH}[9,10]$. The main advantage of using this method is that bioactive agents such as growth factors, enzymes and DNAs, can be co-deposited with CaP crystals onto substrates without losing their bioactivity $[11,12]$. It has been reported that the biomolecule-containing $\mathrm{CaP}$ coating not only improves bone integration with implants, but also induces more new bone formation $[13,14]$.
More recently, researchers have focused on the application of biomimetic $\mathrm{CaP}$ coating as a protein carrier system. Although CaP has been used for protein delivery in the past, most of the therapeutic agents are simply adsorbed onto CaP surfaces [15]. These agents then dissociate from CaP in a "burst release" fashion. The desirable sustained release is not achieved. In comparison, when proteins are co-deposited with CaP crystals in the biomimetic coating process, a sustained protein release is resulted as the coating slowly degrades in vivo $[16,17]$. However, it was found that only a very small portion of the protein incorporated into the coating released out due to the slow degradation rate of most of the CaP coatings at physiological conditions [18-20]. More importantly, the release profile with this approach is not controllable as the release simply relies on the dissolution of the coating.

In the present work, bovine serum albumin (BSA), a protein with similar size of growth factors, was used as a model protein. The distribution of BSA in the biomimetic $\mathrm{CaP}$ coating was carefully tailored by varying the BSA adding time. The BSA release profile and its release effi- 
ciency at different BSA adding times were investigated systemically.

\section{Materials and Methods}

\subsection{Materials}

Commercially available pure titanium strips $(18 \mathrm{~mm} \times 2$ $\mathrm{mm} \times 0.5 \mathrm{~mm}$ ) were used in the current study. They were roughened using $800 \#$ sandpaper, followed by alkaline treatment in $5 \mathrm{M} \mathrm{NaOH}$ at $60^{\circ} \mathrm{C}$ for $24 \mathrm{~h}$. The treated strips were then rinsed thoroughly with de-ionized water and dried at room temperature.

Modified SBF was prepared based on the procedures described by $\mathrm{Qu}$ [21], but the ion concentrations were adjusted according to Table 1. Alexa Fluor 488 conjugate BSA (Alexa488-BSA, Invitrogen, USA) was purchased and used as a model protein in this study.

\subsection{Preparation of Biomimetic Coatings with Different BSA Adding Times}

To avoid protein absorption onto the wall of container, all the tubes used in this study were pre-coated with $1 \%$ non-fluorescent labeled BSA solution by filling the tubes with the BSA solution for 60 minutes, and then washed with PBS for three times. To better tailor the release of BSA, BSA was added to $\mathrm{m}-\mathrm{SBF}$ at different time periods after the coating process started to control the BSA distribution within the coating. Basically, each of the sixteen pretreated titanium strips was vertically placed in a $1.5 \mathrm{~mL}$ tube containing $1.0 \mathrm{~mL} \mathrm{~m}-\mathrm{SBF}$. All the tubes were then incubated in a water bath at $42^{\circ} \mathrm{C}$ for $24 \mathrm{~h}$. BSA was added to each tube after the strip was immersed in m-SBF for 0, 4, 6 and $8 \mathrm{~h}$, respectively. At each interval, four samples were chosen and $26 \mu \mathrm{L}$ Alexa488-BSA at a concentration of $2.0 \mathrm{mg} / \mathrm{mL}$ was added into each tube, resulting in a final BSA concentration of $50 \mu \mathrm{g} / \mathrm{mL}$. After $24 \mathrm{~h}$ incubation in $\mathrm{m}-\mathrm{SBF}$, all the strips were taken out, washed thoroughly with de-ionized water and dried

Table 1. Inorganic composition of human blood plasma and m-SBF.

\begin{tabular}{ccc}
\hline \multirow{2}{*}{ Ion } & \multicolumn{2}{c}{ Concentration $/ \mathrm{mM}$} \\
\cline { 2 - 3 } & Blood plasma & $m-S B F$ \\
\hline $\mathrm{K}^{+}$ & 5.0 & 6.0 \\
$\mathrm{Na}^{+}$ & 142.0 & 110.0 \\
$\mathrm{Ca}^{2+}$ & 2.5 & 8.0 \\
$\mathrm{Mg}^{2+}$ & 1.5 & 1.5 \\
$\mathrm{Cl}^{-}$ & 103.0 & 110.0 \\
$\mathrm{HCO}_{3}{ }^{-}$ & 27.0 & 18 \\
$\mathrm{SO}_{4}{ }^{2-}$ & 0.5 & 0 \\
$\mathrm{HPO}_{4}{ }^{2-}$ & 1.0 & 3.0 \\
\hline
\end{tabular}

at room temperature.

\subsection{Characterization of Biomimetic Coatings Prepared with Different BSA Adding Time}

The morphology of the coatings prepared at different BSA adding times was observed using field emission scanning electron microscopy (FESEM, JEOL 6335F, Japan). The coatings were also examined using X-ray diffractometer (Bruker AXS D5005) with a copper target. The voltage and current setup were $40 \mathrm{kV}$ and $40 \mathrm{~mA}$, respectively. A step size of $0.02^{\circ}$ and a scan speed of $0.5^{\circ} / \mathrm{min}$ were used. Infrared spectra of the coatings were recorded by Fourier transform infrared (FTIR) spectroscopy (Nicolet XS60). The coatings were removed gently from substrate using a knife and ground into powder for FTIR examination. FTIR spectra were obtained between wave numbers $4000-400 \mathrm{~cm}^{-1}$ at a resolution of $2 \mathrm{~cm}^{-1}$ and an average scan of 128.

\subsection{Incorporation of BSA, Calcium, and Phosphate into Bbiomimetic Coatings}

The BSA concentration in the remained m-SBF was measured using a microplate reader (Molecular Devices M2 plate reader, USA) at a fluorescence absorbance mode with an excitation wavelength of $497 \mathrm{~nm}$ and emission wavelength of $520 \mathrm{~nm}$. The incorporation efficiency (in percentage) of BSA incorporated into the coating was calculated using the following equation:

$$
\mathrm{E}_{\text {incorporation }}(\%)=\frac{\left(\mathrm{C}_{\text {initial }}-\mathrm{C}_{\text {remained }}\right)}{\mathrm{C}_{\text {initial }}} \times 100 \%
$$

where $\mathrm{E}_{\text {incorporation }}$ BSA incorporation efficiency in the coating;

$\mathrm{C}_{\text {initial }}$ : Initial BSA concentration in m-SBF (amount pre-determined);

$\mathrm{C}_{\text {remained: }}$ BSA concentration remained in m-SBF after the coating.

The amount of calcium and phosphate in m-SBF participating in the coating formation was also calculated using a similar equation. The calcium concentration in the remaining $\mathrm{m}-\mathrm{SBF}$ was measured using an atomic absorbance spectrometer (AAS, Perkin Elmer-5000, USA), and the phosphate concentration was determined using a molybdenum blue chemistry method. Briefly, pure water, 2.5\% ammonium molybdate, and $10 \mathrm{wt} \%$ ascorbic acid $(\mathrm{v}: \mathrm{v}: \mathrm{v}=5: 1: 1)$ were added in the above order to form $\mathrm{a}$ working solution. The working solution was further mixed with the testing solution at a volume ratio of $4: 1(\mathrm{v} / \mathrm{v})$, and then incubated at $60^{\circ} \mathrm{C}$ for $15 \mathrm{~min}$. The mixed solution was subsequently examined using a microplate reader (Biotek, MQX200) at a wavelength of $830 \mathrm{~nm}$. 


\subsection{BSA Release Behavior in Vitro from Biomimetic Coatings Prepared with Different BSA Adding Time}

To study the effect of BSA adding time on BSA release behavior, the biomimetic apatite coated titanium strips were further employed for the release study. The strips with different Alexa488-BSA adding times $(\mathrm{n}=4)$ were placed in a sealed $1.5 \mathrm{~mL}$ tube containing $1.0 \mathrm{~mL}$ phosphate buffered saline (PBS, $\mathrm{pH}=7.4$ ) and kept at $37^{\circ} \mathrm{C}$ in a dynamic water bath. All soaking media were removed from the tube and saved for Alexa488-BSA concentration determination at 1, 2, 4, 8, 12 and $24 \mathrm{~h}$ for the first 24 hours. Fresh PBS of $1.0 \mathrm{~mL}$ was refilled to the tube to maintain a constant volume of the soaking medium. After that, the soaking medium was assessed every $24 \mathrm{~h}$ up to 240 h. The concentration of Alexa488-BSA was measured as stated in Section 2.4 .

\subsection{Degradation Study of the Coatings Prepared with Different BSA Adding Times}

After the release study, the strips were collected and rinsed with de-ionized water thoroughly and dried at room temperature overnight. The morphology of the remaining coatings was observed using a FESEM. To quantify the degradation rate, the coatings after the release study were dissolved in $1.0 \mathrm{~mL} \mathrm{HCl}(1.0 \mathrm{M})$ and the supernatants were collected for calcium and phosphate quantifications. Calcium and phosphate amounts remained in the coating after the release study were measured using the same method described in Section 2.4.

\section{Results}

\subsection{Characterization of Biomimetic Coatings Prepared with Different BSA Adding Times}

FESEM observation indicates that the surface morphology of the coatings changed significantly with different BSA adding times. Homogenous, porous coatings were deposited on all titanium strip surfaces regardless of the BSA adding time. These coatings demonstrated morphology of long sharp interconnected crystal plates covered by a leaf-like material. The later the BSA was added to the coating, the more leaf-like material observed on the surface of the coating (Figure 1). When BSA was added at the initial stage of the coating (e.g. $0 \mathrm{~h}$ adding time), a porous uniform coating was observed with the crystal plates slightly bended (Figures 1(a), (b)). No leaf-like material was observed for this group of samples. The leaf-like material started to appear as BSA was added $4 \mathrm{~h}$ after the coating process (Figures 1(c), (d)). When BSA was added to m-SBF $6 \mathrm{~h}$ after soaking, nearly half of the coating was covered by the leaf-like material (Figures 1(e), (f)). Much more leaf-like materials was ob-
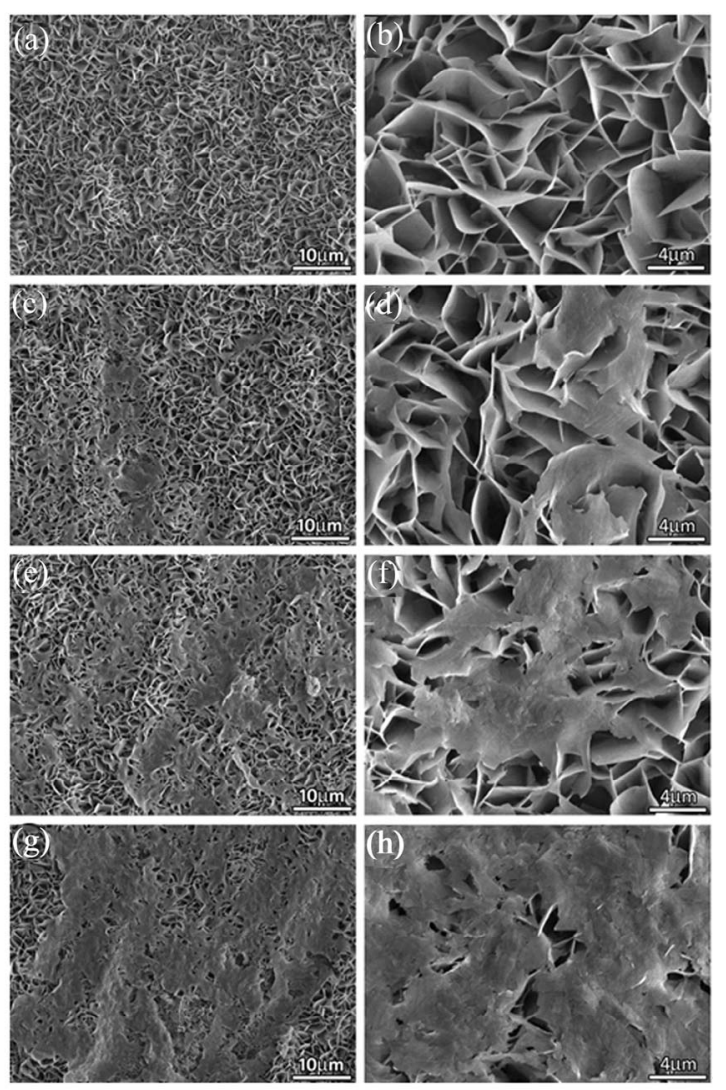

Figure 1. SEM micrographs of CaP coatings soaked in $\mathbf{m}-$ SBF containing $50 \mu \mathrm{g} / \mathrm{mL}$ BSA: BSA was added into $\mathrm{m}-\mathrm{SBF}$ after the coating process proceeded for $0,4,6$ and $8 \mathrm{~h}$, respectively. (a) 0 h, $\times 2000$ (b) 0 h, $\times 10000$ (c) 4 h, $\times 2000$ (d) $4 \mathrm{~h}, \times 10000$ (e) 6 h, $\times 2000$ (f) 6 h, $\times 10000$ (g) 8 h, $\times 2000$ (h) $8 \mathrm{~h}, \times 10000$.

served when BSA was added $8 \mathrm{~h}$ after soaking (Figures 1(g), (h)).

Under a higher magnification, it was noted that the plate-like mineral layer had been formed under the leaflike material.

The biomimetic coatings were also characterized by FTIR spectroscopy and XRD analysis. As shown in Figure 2(a), the XRD patterns indicated that the biomimetic coating is a typical apatite phase. The peaks around $31-33^{\circ}$ is an overlap of three major peaks of apatite: (211), (112) and (300). The sharp peak at $26^{\circ}$ is assigned to the $\mathrm{CaP}$ coating formed with a strong preferential crystallographic direction of (002). No considerable differences in those characteristic diffraction peaks were discerned from the coatings prepared by adding BSA at different time periods. The FTIR spectra of all CaP coatings with different BSA adding times show phosphate absorption bands at 1031, 600, and $561 \mathrm{~cm}^{-1}$ (Figure 2(b)). An amide peak is shown in all four coatings at $1652 \mathrm{~cm}^{-1}$ while another low amide peak was only detected at $1539 \mathrm{~cm}^{-1}$ 


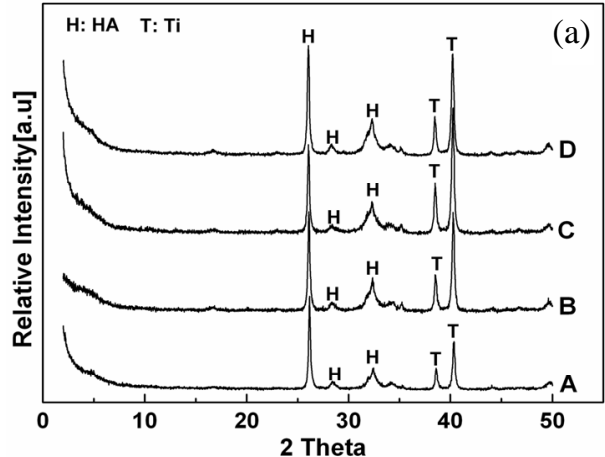

(a)

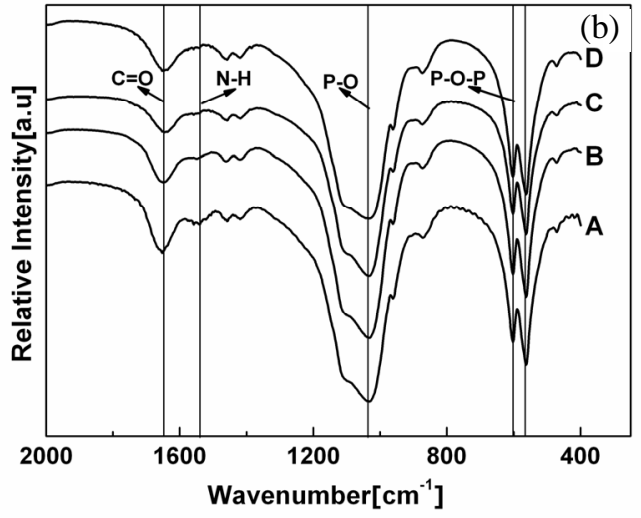

(b)

Figure 2. Chemical and crystalline structure of CaP coatings soaked in $\mathrm{m}-\mathrm{SBF}$ containing $50 \mu \mathrm{g} / \mathrm{mL}$ BSA versus soaking time. (a) XRD patterns of $\mathrm{CaP}$ coatings with different BSA adding times: (A) 0 h, (B) $4 \mathrm{~h}$, (C) $6 \mathrm{~h}$, (D) $8 \mathrm{~h}$; and (b) FTIR spectra of CaP coatings with different BSA adding times: (A) 0 h, (B) 4 h, (C) 6 h, (D) 8 h.

in the coatings with earlier BSA adding times, such as 0 $\mathrm{h}$ and $4 \mathrm{~h}$. The absence of the $1539 \mathrm{~cm}^{-1}$ peak in the coatings with a BSA adding time of $6 \mathrm{~h}$ and $8 \mathrm{~h}$ might due to their lower BSA incorporation efficiencies comparing to those with a BSA adding time of $0 \mathrm{~h}$ and $4 \mathrm{~h}$.

\subsection{The Effect of BSA Adding Time on the Incorporation Efficiency of BSA, Calcium and Phosphate}

Figure 3 shows the incorporation efficiency of BSA, calcium and phosphate at different BSA adding times. It was found that the BSA incorporation efficiency decreased dramatically as the BSA adding time was delayed. When BSA was added to m-SBF after $4 \mathrm{~h}$ of soaking, its incorporation efficiency was hardly affected. An incorporation efficiency of approximately 75\% was achieved. However, BSA incorporation efficiency dropped sharply to $55 \%$ when the adding time was delayed from $4 \mathrm{~h}$ to $6 \mathrm{~h}$ of soaking. When BSA was added at $8 \mathrm{~h}$ after soaking, only $26 \%$ BSA in the m-SBF was incorporated into the coating. In contrast, the incorpo-

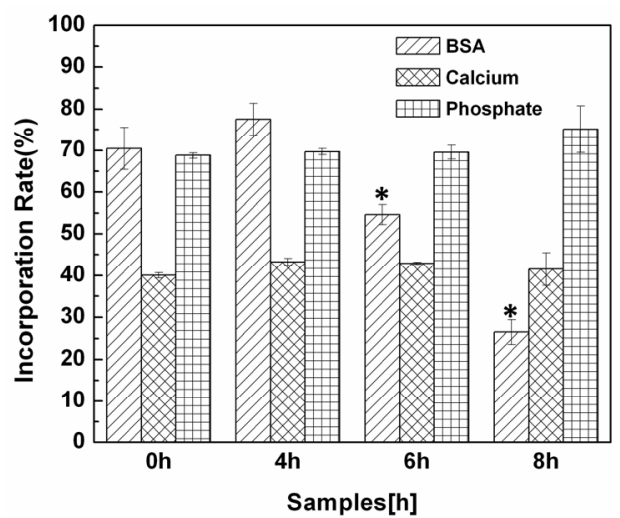

Figure 3. Incorporation efficiencies of BSA, calcium and phosphate versus BSA adding times: BSA was added into $\mathrm{m}$-SBF after $0 \mathrm{~h}, 4 \mathrm{~h}, 6 \mathrm{~h}$ and $8 \mathrm{~h}$ of samples soaked in m-SBF.

ration of calcium and phosphate in $\mathrm{m}-\mathrm{SBF}$ was not affected by the change of the BSA adding time. About $40 \%$ of calcium and $70 \%$ of phosphate in m-SBF were participated the calcium phosphate coating formation regardless of the change of BSA adding time.

\subsection{BSA Release Behavior from Biomimetic Coatings Prepared with Different BSA Adding Time in Vitro}

The BSA release profiles at different BSA adding times are shown in Figure 4. Although a "bursting release" is shown at the initial stage, a sustained release was subsequently observed, suggesting a typical two-stage release mechanism when the BSA adding times were 0,4 , and 6 h. However, when BSA was added $8 \mathrm{~h}$ after soaking, a more pronounced "burst release" at the initial stage was observed, which resulted in the release of more than $80 \%$ of the BSA incorporated into the coating. The total amount of BSA released from the coatings with BSA adding times at 0,4 , and $6 \mathrm{~h}$ are similar, approximately $7.5 \mu \mathrm{g}$, but a dramatic drop was observed for the coating with the BSA adding time at $8 \mathrm{~h}$ (Figure 4(a)). The percentage of the incorporated BSA released with time was also calculated (Figure $\mathbf{4 ( b ) )}$ ). When BSA was added to $\mathrm{m}$-SBF at the very beginning of the soaking, the percentage of the BSA released was very low. Only $22 \%$ of the BSA incorporated into the coating was released after 10 days of soaking. In comparison, the percentage of BSA release was much higher for the coating with BSA added at 4 and $6 \mathrm{~h}$ after soaking, 28\% and 32\% respectively.

\subsection{Degradation Study of the Coatings Prepared with Different BSA Adding Time}

To study the coatings degradation, calcium and phosphate amounts of the remaining coating after 10 day of 

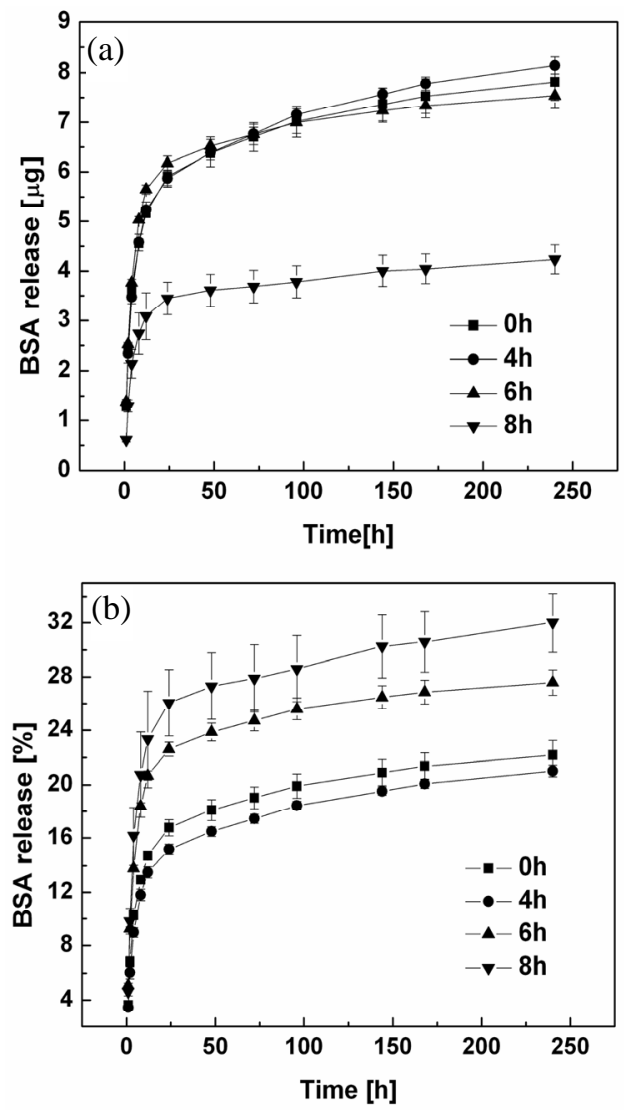

Figure 4. BSA release profiles from CaP coatings prepared with different BSA adding time: (a) The amount of BSA released from CaP coatings. (b) The percentage of BSA released from CaP coatings. BSA was added to $\mathrm{m}$-SBF after the coating process proceeded for $0,4,6$ and $8 \mathrm{~h}$, respectively.

soaking were measured and the results are demonstrated in Figure 5. It was found that the BSA adding time had a distinct influence on the coating degradation rate. When BSA was added at the beginning of the coating (adding time at $0 \mathrm{~h}$ ), about $28 \%$ calcium and $17 \%$ phosphate were dissolved during the 10-day soaking. The amount of degraded calcium increased to approximately $35 \%$ and phosphate to $25 \%$ for both the adding time of 4 and $6 \mathrm{~h}$. It is worth to notice that the coating prepared with BSA adding time at $8 \mathrm{~h}$ had the slowest degradation rate, 20\% for calcium and $14 \%$ for phosphate.

The morphology of the coatings changed dramatically after the 10-day of BSA release study, as shown in Figure 6. The leaf-like material presented on the surface of most of the coatings disappeared completely after the 10day release study. In turn, porous, homogenous coatings were observed. It was also discovered that the large plate-like mineral layer, which was found beneath the leaf-like material after $24 \mathrm{~h}$ of soaking in m-SBF, also disappeared. Instead, a coating with small pores was ma-

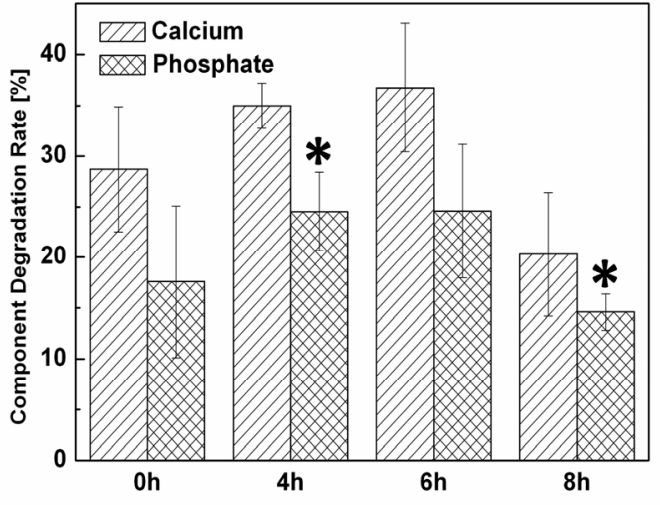

Figure 5. Degradation of CaP coatings after 10 days immersion in PBS. The degradation of both calcium and phosphate was calculated based on the amount of calcium and phosphate in coatings before and after the release study. BSA was added to $\mathrm{m}$-SBF after the coating process proceeded for $0,4,6$ and 8 h, respectively.
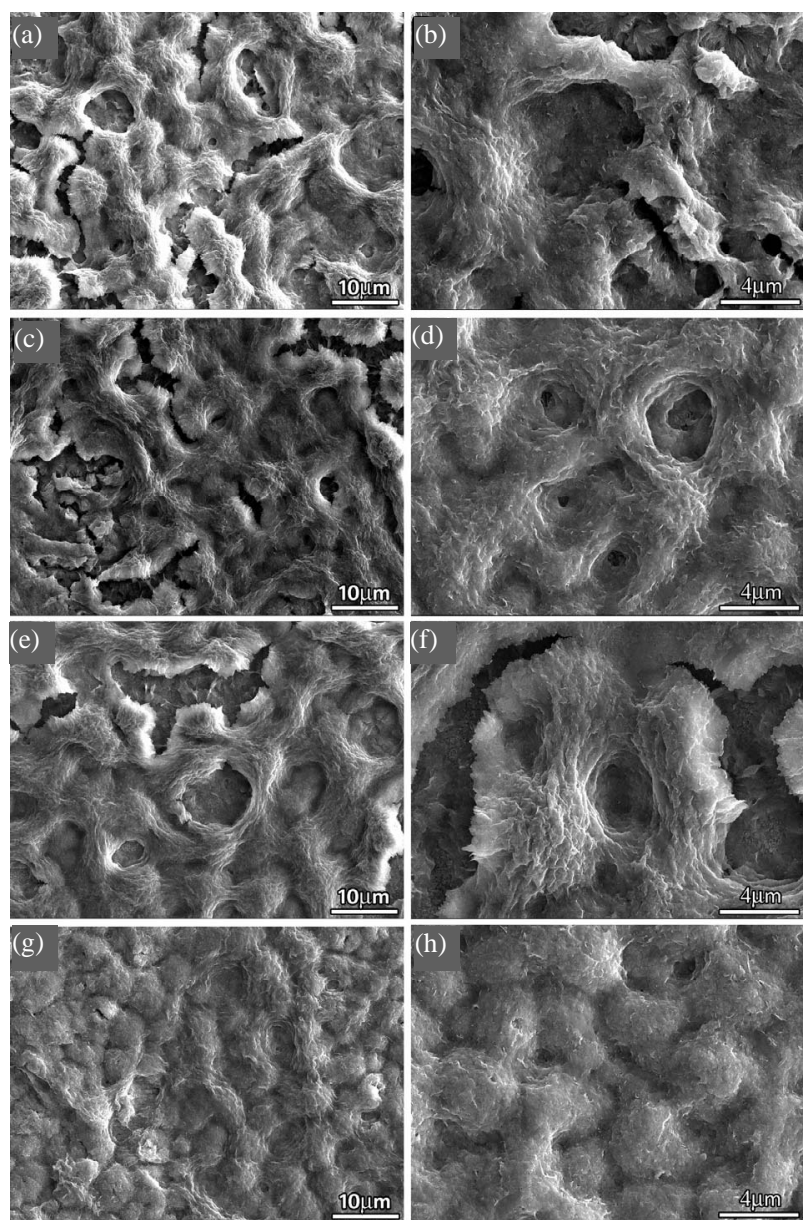

Figure 6. SEM micrographs of CaP coatings after $10 \mathrm{~d}$ in vitro BSA release study: BSA was added to $\mathrm{m}-\mathrm{SBF}$ after the coating process proceeded for $0,4,6$ and $8 \mathrm{~h}$, respectively. (a) 0 h, $\times 2000$ (b) 0 h, $\times 5000$ (c) 4 h, $\times 2000$ (d) 4 h, $\times 5000$ (e) 6 h, $\times 2000$ (f) 6 h, $\times 5000$ (g) 8 h, $\times 2000$ (h) 8 h, $\times 5000$. 
nifested (Figures 6(a)-(f)), which was similar to that of the coating formed after $6 \mathrm{~h}$ soaking in m-SBF (data not shown). In addition, the morphology of the coating with BSA adding time at $8 \mathrm{~h}$ was slightly different from those with BSA added at an earlier stage (Figure 6(g), (h)). No small pores were observed.

\section{Discussion}

Biomimetic calcium phosphate coatings have been proved to be a suitable biomolecule carrier [19,22-24]. Different biomolecules, such as growth factors, antibiotics, enzymes and DNAs, have been successfully incorporated into CaP coatings in recent years [12,14,25]. However, most of these applications suffer from both low bimolecular incorporation efficiency and low release efficiency. In addition, the release behavior of these biomolecules from CaP coatings is far from satisfaction [18,19-20]. In our previous study, we managed to improve the BSA incorporation efficiency up to $90 \%$ by closely controlling the ratio of $\mathrm{m}-\mathrm{SBF}$ solution volume to sample surface area [26]. In the present study, we tailored the release behavior of BSA by simply delaying the BSA adding time to the m-SBF. Our results demonstrated that the carrier system has a high protein incorporation rate, improved release efficiency and sustained release profile when the BSA adding time is $6 \mathrm{~h}$.

To control the release profile of BSA from CaP coating, it is crucial to develop a better understanding of the mechanism of BSA incorporation into the CaP coating. Our results (data not shown) indicated that most of the BSA incorporated into the CaP coating in the initial 4-12 $\mathrm{h}$ soaking in m-SBF. Moreover, the BSA incorporation profile perfectly matched with the incorporation profiles of calcium and phosphate during the initial 4-12 h. As a result, it is believed that $\mathrm{BSA}$ is attached to the $\mathrm{CaP}$ and co-deposited onto the strip. When it is added to the $\mathrm{m}-\mathrm{SBF}$ at the beginning of the coating, BSA is homogenously distributed within the entire depth of the coating, as illustrated in Figure 7(a). However, when BSA is added after a certain time period after the coating starts, it only presents in the top section of the coating (Figure 7(b)). Such incorporated BSA would be easier to release compared to those embedded in the bottom coating adjacent to the substrate. To test this hypothesis, we have managed to retain all the BSA to the top section of the coating by delaying the BSA adding time. One advantage of this method is that we can easily control the BSA distribution within the coating, which in turn controls the protein release from the coatings. Another advantage of the method is that the amount of BSA released from the coating is substantially increased as less BSA is trapped in the coating comparing to the conventional approach.

By adjusting the BSA adding time, variable release curves with different release efficiency was attained, which indicated that varying BSA adding time can be an efficient approach to control the release of biomolecules from the biomimetic CaP coating (Figure 4). It was also found that the BSA release amount was inversely proportional to BSA adding time, but the release efficiency was proportional to BSA adding time (Figure 4(b)). This might be explained as follows: Since BSA is co-precipitated with $\mathrm{CaP}$ coating on the substrate, it is distributed evenly through the entire depth of the coating when it is added at 0 h (Figure 7). However, the bottom section of the coating hardly degrades, which results in a relatively large amount of protein is still trapped in the coating after the 10 -day release test $[27,28]$. As a result, low release efficiency was attained for the coating with an adding time of $0 \mathrm{~h}$. When the adding time is increased, the BSA is only distributed in the top section of the coating which makes it easier for BSA to release from the coating via degradation. In the case of adding time at $8 \mathrm{~h}$, the majority of BSA was simply adsorbed onto the surface of the coating, so an stronger initial bursting release was observed due to the desorption of BSA from the coating. The results of coating degradation study are also consistent with this explanation. It is known that biomimetic CaP coating can be gradually dissolved under physiological conditions [29]. The dissolution rate of CaP is affected by its crystallinity, composition, and porosity [27]. It was observed that only the top layer of the coatings was dissolved after $10 \mathrm{~d}$ release study, which suggests only the BSA in this part of the coating be released. It is worth to note that high release efficiency is achieved,

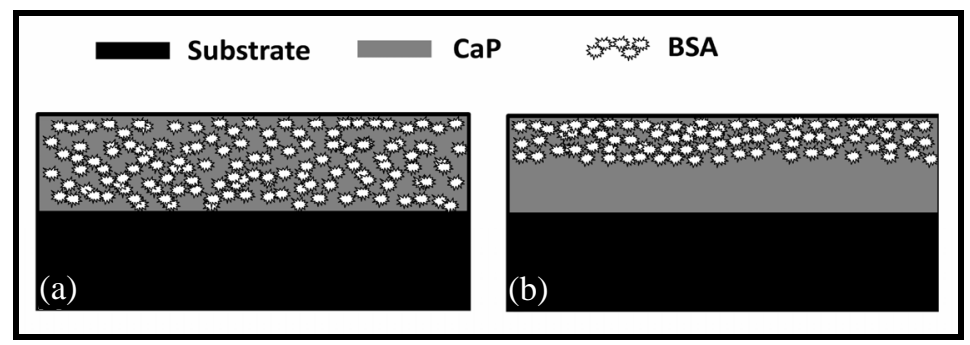

Figure 7. Schematic illustration of BSA distribution in CaP coatings (a) BSA is added to m-SBF from the beginning of the coating process. (b)BSA is added to $\mathrm{m}$-SBF after the coating process proceeded for certain time period. 
but it is accompanied with lower protein incorporation efficiency. According to the release data, adding BSA to $\mathrm{m}$-SBF at $6 \mathrm{~h}$ after soaking is an optimal time to achieve both high incorporation rate and release efficiency.

BSA adding time also significantly affects BSA incorporation efficiency into/onto the biomimetic coating. It was observed that the BSA incorporation efficiency decreased nearly linearly as a function of BSA adding time (Figure 3). It is known that the BSA incorporation efficiency is closely associated with calcium and phosphate precipitation rate. Since the incorporation efficiency of calcium and phosphate remains around $40 \%$ and $70 \%$ respectively regardless of the BSA adding time, the BSA incorporation efficiency is mainly determined by the time when BSA is added to the system. The later the BSA is added, the less the $\mathrm{Ca}^{2+}$ ions are available to bind to BSA and co-precipitate onto the substrate surface, and the lower the BSA incorporation efficiency.

Especially, in the case of the adding time at $8 \mathrm{~h}$, the BSA incorporation relied mainly on pseudo-Langmuir adsorption as the majority of the coating had been formed [30]. In contrast to BSA incorporation efficiency, the incorporation of calcium and phosphate was not affected by BSA adding time (Figure 3). Besides, the XRD and FTIR results also suggest that there is no significant change in the coating composition by varying the BSA adding time. Thus, changing BSA adding time during coating formation not only provides better control over release profiles, but also reserves all the properties of the biomimetic CaP coating, such as coating thickness, composition, and crystallinity.

\section{Conclusion}

In this study, we have successfully optimized the release profile of BSA from CaP coating by adjusting its adding time. At a BSA adding time of $6 \mathrm{~h}$, its release efficiency was improved to $26 \%$ while its incorporation efficiency was maintained as high as $55 \%$. In this study, BSA was employed as a model protein. The method established here, however, can be applied for loading other drugs or proteins using biomimetic $\mathrm{CaP}$ coatings as a carrier.

\section{Acknowledgements}

The authors would like to thank the supports from National Science Foundation (DMI 0500269 and BES 0503315) and Connecticut Innovations under the Yankee Ingenuity Technology Competition. The authors would also like to thank Prof. Yong Wang in the Department of Chemical, Material and Biomolecular Engineering, University of Connecticut for helpful discussion.

\section{REFERENCES}

[1] R. Narayanan, S. K. Seshadri, T. Y. Kwon and K. H. Kim,
"Calcium Phosphate-Based Coatings on Titanium and Its Alloys,” Journal of Biomedical Materials Research: Part B-Applied Biomaterials, Vol. 85B, No. 1, 2008, pp. 279299. doi:10.1002/jbm.b.30932

[2] R. Z. LeGeros, "Properties of Osteoconductive Biomaterials: Calcium phosphates," Clinical Orthopaedics and Related Research, Vol. 395, 2002, pp. 81-98. doi:10.1097/00003086-200202000-00009

[3] J. Weng, M. Wang and J. Y. Chen, "Plasma-Sprayed Calcium Phosphate Particles with High Bioactivity and Their Use in Bioactive Scaffolds,” Biomaterials, Vol. 23, No. 13, 2002, pp. 2623-2629. doi:10.1016/S0142-9612(01)00393-3

[4] J. G. C. Wolke, K. de Groot and J. A. Jansen, "In vivo Dissolution Behavior of Various RF Magnetron Sputtered Ca-P Coatings,” Journal of Biomedical Materials Research, Vol. 39, No. 4, 1998, pp. 524-530.

[5] E. Milella, F. Cosentino, A. Licciulli and C. Massaro, "Preparation and Characterisation of Titania/Hydroxyapatite Composite Coatings Obtained by Sol-Gel Process," Biomaterials, Vol. 22, No. 11, 2001, pp. 1425-1431. doi:10.1016/S0142-9612(00)00300-8

[6] F. Barrere, P. Layrolle, C. A. van Blitterswijk and K. de Groot, "Biomimetic Coatings on Titanium: A Crystal Growth Study of Octacalcium Phosphate," Journal of Materials Science: Materials in Medicine, Vol. 12, No. 6, 2001, pp. 529-534. doi:10.1023/A:1011271713758

[7] F. Barrere, P. Layrolle, C. A. van Blitterswijk, K. de Groot, "Biomimetic Calcium Phosphate Coatings on Ti6AI4V: A Crystal Growth Study of Octacalcium Phosphate and Inhibition by Mg2+ and HCO3,” Bone, Vol. 25, No. 2, 1999, pp. 107S-111S.

[8] W.-Q. Yan, T. Nakamura, K. Kawanabe, S. Nishigochi, M. Oka and T. Kokubo, "Apatite Layer-Coated Titanium for Use as Bone Bonding Implants,” Biomaterials, Vol. 18, No. 17, 1997, pp. 1185-1190. doi:10.1016/S0142-9612(97)00057-4

[9] P. J. Li, C. Ohtsuki, T. Kokubo, K. Nakanishi, N. Soga and K. Degroot, "The Role of Hydrated Silica, Titania, and Alumina in Inducing Apatite on Implants,” Journal of Biomedical Materials Research, Vol. 28, No. 1, 1994, pp. 7-15. doi:10.1002/jbm.820280103

[10] T. Kokubo and H. Takadama, "How Useful is SBF in Predicting in Vivo Bone Bioactivity?” Biomaterials, Vol. 27, No. 15, 2006, pp. 2907-2915.

[11] Y. Liu, L. Enggist, A. F. Kuffer, D. Buser and E. B. Hunziker, "The Influence of BMP-2 and Its Mode of Delivery on The Osteoconductivity of Implant Surfaces During the Early Phase of Osseointegration,” Biomaterials, Vol. 28, No. 16, 2007, pp. 2677-2686. doi:10.1016/j.biomaterials.2007.02.003

[12] H. Shen, J. Tan and W. M. Saltzman, "Surface-Mediated Gene Transfer from Nanocomposites of Controlled Texture,” Nature Materials, Vol. 3, No. 8, 2004, pp. 569-574

[13] Y. Liu, E. B. Hunziker, N. X. Randall, K. de Groot and P. Layrolle, "Proteins Incorporated into Biomimetically Prepared Calcium Phosphate Coatings Modulate Their 
Mechanical Strength and Dissolution Rate,” Biomaterials, Vol. 24, No. 1, 2003, pp. 65-70.

[14] Y. Liu, R. O. Huse, K. de Groot, D. Buser and E. B. Hunziker, "Delivery Mode and Efficacy of BMP-2 in Association with Implants," Journal of Dental Research, Vol. 86, No. 1, 2007, pp. 84-89. doi:10.1177/154405910708600114

[15] P. Laffargue, P. Fialdes, P. Frayssinet, M. Rtaimate, H. F. Hildebrand and X. Marchandise, “Adsorption and Release of Insulin-Like Growth Factor-I on Porous Tricalcium Phosphate Implant," Journal of Biomedical Materials Research, Vol. 49, No. 3, 2000, pp. 415-421.

[16] Y. Liu, J. P. Li, E. B. Hunziker and K. de Groot, "Incorporation of Growth Factors into Medical Devices via Biomimetic Coatings," Philosophical Transactions of the Royal Society a-Mathematical Physical and Engineering Sciences, Vol. 364, No. 1838, 2006, pp. 233-248.

[17] T. Y. Liu, S. Y. Chen, D. M. Liu and S. C. Liou, "On the Study of BSA-Loaded Calcium-Deficient Hydroxyapatite Nano-Carriers for Controlled Drug Delivery,” Journal of Control Release, Vol. 107, No. 1, 2005, pp. 112-121. doi:10.1016/j.jconrel.2005.05.025

[18] Y. Liu, P. Layrolle, J. de Bruijn, C. van Blitterswijk and K. de Groot, "Biomimetic Coprecipitation of Calcium Phosphate and Bovine Serum Albumin on Titanium Alloy," Journal of Biomedical Materials Research, Vol. 57, No. 3, 2001, pp. 327-335.

[19] C. Du, G. B. Schneider, R. Zaharias, C. Abbott, D. Seabold, C. Stanford and J. Moradian-Oldak, "Apatite/ Amelogenin Coating on Titanium Promotes Osteogenic Gene Expression,” Journal of Dental Research, Vol. 84, No. 11, 2005, pp. 1070-1074. doi:10.1177/154405910508401120

[20] H. B. Wen, J. R. de Wijn, C. A. van Blitterswijk and K. de Groot, "Incorporation of Bovine Serum Albumin in Calcium Phosphate Coating on Titanium," The Journal of Biomedical Materials Research, Vol. 46, No. 2, 1999, pp. 245-252.

[21] H. Qu and M. Wei, "The Effect of Temperature and Initial $\mathrm{Ph}$ on Biomimetic Apatite Coating," Journal of Biomedical Materials Research-Part B Applied Biomaterials, Vol. 87, No. 1, 2008, pp. 204-212. doi:10.1002/jbm.b.31096

[22] H. S. Azevedo, I. B. Leonor, C. M. Alves and R. L. Reis, "Incorporation of Proteins and Enzymes at Different Stages of the Preparation of Calcium Phosphate Coatings on a Degradable Substrate by a Biomimetic Methodology," Materials Science and Engineering: C, Vol. 25, No. 2, 2005, pp. 169-179.

[23] M. Stigter, J. Bezemer, K. de Groot and P. Layrolle, "Incorporation of Different Antibiotics into Carbonated Hydroxyapatite Coatings on Titanium Implants, Release and Antibiotic Efficacy,” Journal of Controlled Release, Vol. 99, No. 1, 2004, pp. 127-137. doi:10.1016/j.jconrel.2004.06.011

[24] M. Stigter, K. de Groot and P. Layrolle, “Incorporation of Tobramycin into Biomimetic Hydroxyapatite Coating on Titanium," Biomaterials, Vol. 23, No. 20, 2002, pp. 4143-4153. doi:10.1016/S0142-9612(02)00157-6

[25] A. Oyane, Y. Yokoyama, M. Uchida and A. Ito, "The Formation of an Antibacterial Agent-Apatite Composite Coating on a Polymer Surface Using a Metastable Calcium Phosphate Solution,” Biomaterials, Vol. 27, No. 17, 2006, pp. 3295-3303. doi:10.1016/j.biomaterials.2006.01.029

[26] X. Yu, H. Qu, D. Knecht and M. Wei, "Incorporation of Bovine Serum Albumin into Biomimetic Coatings on Titanium with High Loading Efficacy and Its Release Behavior,” Journal of Materials Science: Materials in Medicine, Vol. 20, No. 1, 2009, pp. 287-294. doi:10.1007/s10856-008-3571-6

[27] F. Barrere, C. M. van der Valk, R. A. Dalmeijer, C. A. van Blitterswijk, K. de Groot and P. Layrolle, "In vitro and in vivo Degradation of Biomimetic Octacalcium Phosphate and Carbonate Apatite Coatings on Titanium Implants," The Journal of Biomedical Materials Research A, Vol. 64, No. 2, 2003, pp. 378-387.

[28] P. Ducheyne and Q. Qiu, "Bioactive Ceramics: The Effect of Surface Reactivity on Bone Formation and Bone Cell Function,” Biomaterials, Vol. 20, No. 23-24, 1999, pp. 2287-2303. doi:10.1016/S0142-9612(99)00181-7

[29] Z. J. Wu, B. Feng, J. Weng, S. X. Qu, J. X. Wang and X. Lu, "Biomimetic Apatite Coatings on Titanium Coprecipitated with Cephradine and Salviae Miltlorrhizae," Journal of Biomedical Materials Research Part B-Applied Biomaterials, Vol. 84B, No. 2, 2008, pp. 486-492. doi:10.1002/jbm.b.30895

[30] K. Kandori, M. Saito, H. Saito, A. Yasukawa and T. Ishikawa, "Adsorption of Protein on Nonstoichiometric Calcium Strontium Hydroxyapatite," Colloids and Surfaces a-Physicochemical and Engineering Aspects, Vol. 94, No. 2-3, 1995, pp. 225-230. 